\title{
Mejoras prácticas para líneas de decoración Inkjet en cerámica. Máquina IR de radiaciones electromagnéticas (tecnología de longitud de onda)
}

\author{
J. GÁLVEZ, D. GÁLVEZ \\ SACMI IBÉRICA, S.A. Gran Vía Tárrega Monteblanco, 263. 12006 Castellón
}

Este trabajo ha sido premiado con el Alfa de Oro en la Feria Internacional de Cerámica de Valencia CEVISAMA 2012

\begin{abstract}
SACMI IBÉRICA, S.A., ha sido galardonada por la Sociedad Española de Cerámica y Vidrio (S.E.C.V.), con un ALFA DE ORO, en su edición 2012, durante la celebración de CEVISAMA, por la presentación de la innovadora Máquina IR de Radiaciones Electromagnéticas, que mejora las condiciones y rendimiento productivo de las líneas de decoración digital INKJET y también en otras aplicaciones decorativas en azulejos y baldosas cerámicas.
\end{abstract}

Palabras clave: Decoración digital Inkjet; Nitidez de diseño; Limpieza de inyectores; Longitud de onda; Energía radiante intensa y rápida

Best practices for inkjet decoration lines in ceramics. Electromagnetic radiation IR machine (wavelength technology)

SACMI IBÉRICA, S.A., has been awarded by the Spanish Society of Ceramics and Glass (SECV), with one GOLD ALFA, in its 2012 edition, during CEVISAMA for the presentation of the innovative IR Electromagnetic Radiation Machine, which improves the conditions and the production performance of digital decoration lines INKJET and other decorative applications ceramic tile.

Keywords: Digital Inkjet Decoration, Design Sharpness, Cleaning injectors, Wavelength, Intense radiant energy and fast.

\section{INTRODUCCIÓN}

SACMI IBÉRICA es una empresa española, perteneciente al Grupo SACMI (Italia), que trabaja en el sector cerámico español desde hace décadas. Contribuye al desarrollo del sector aportando en cada momento, la tecnología y máquinas más avanzadas para permitir la producción de azulejos, baldosas y placas cerámicas, de primerísima calidad, que son muy apreciadas en los mercados de todo el mundo.

Además de nuevas máquinas y hornos, se estudian sistemas y equipos que permitan, en fábricas ya existentes, reducir los consumos de energía; aumento de productividad de las líneas de fabricación y mejoras en la calidad del producto.

Un reciente acuerdo de colaboración con BULMA TECNOLOGÍA, S.L., que es la empresa poseedora de la patente P200702733ES, nos ha permitido la presentación de esta innovadora máquina IR en CEVISAMA 2012.

\section{ANTECEDENTES Y NECESIDAD DE LA MÁQUINA IR DE RADIACIONES}

Con la aplicación industrial de las Máquinas de Decoración Digital, se están detectando algunos problemas que dificultan el buen rendimiento y resultado de ésta interesantísima tecnología: a) Un problema que afecta a la calidad del diseño es debido a que, en ocasiones, un producto fabricado meses antes con buen resultado, no mantiene la nitidez de sus líneas al ponerse nuevamente en producción. Las gotas de tinta se difuminan en la superficie decorada.

Normalmente, los usuarios piensan que ésta peor definición del diseño es debida a pequeñas variaciones de las características de los esmaltes (tintas), entre las dos partidas de fabricación, viscosidad, etc.

b) Otro problema importante en la producción con Decoradoras Digitales, es la frecuente necesidad de limpieza de los inyectores.

La evaporación del agua que posee la baldosa cerámica, a su paso bajo el cabezal de impresión, genera condensaciones que obstruyen los inyectores. Hay que tener presente también que en el sistema de impresión D.O.D. el paso de agua si condensase en el interior, produciría la parada del cabezal de la impresora.

La limpieza de los inyectores obligan a interrumpir frecuentemente la producción de la línea de esmaltado/ decoración, pudiendo llegar a ser de 15 min. aprox. en ocasiones, según productos. 
Este problema se imputa a carencias de la máquina decoradora, y a inconstancia en los esmaltes y se intenta resolver instalando grandes cabinas cerradas con aire inyectado o soplantes de aire caliente o frío. Dichas aplicaciones mejoran algo los resultados, pero sin llegar a resolver el problema en grado satisfactorio.

\section{ANÁLISIS DEL PROCESO ANTERIOR A LA DECORA- CIÓN DIGITAL}

Después de razonar sobre estos hechos, pensamos que ambos problemas están relacionados con la excesiva humedad superficial de las baldosas, que en algunas condiciones rebasa los límites admitidos por el proceso de producción para obtener una buena definición del diseño, calidad del producto y aumenta el proceso de condensaciones dentro de la máquina.

\section{OBJETIVOS A CONSEGUIR}

- Reducir los vapores y humedad superficial, en las piezas y con ello reducir las condensaciones en inyectores y otros elementos de la decoradora.

- Evitar paradas de producción para conseguir una mayor productividad de la línea de decoración.

- Evitar problemas de difusión de las gotas de tinta, en la superficie de la baldosa mejorando la calidad estética del producto y con ello revalorizar el producto.

\section{SOLUCIÓN A LOS PROBLEMAS DESCRITOS}

La solución más favorable debe ser eliminar el agua en un estrato del material momentos antes de la decoración digital, aplicando energía térmica de gran intensidad y rapidez mediante un sistema automático y sencillo de utilización con un consumo de energía durante los momentos de trabajo y sinconsumo en las pausas de producción.

ESQUEMA DE UTILIZACION DE LA MAQUINA I.R. DE RADIACIONES ELECTROMAGNETICAS DE LONGUITUD DE ONDA, SOBRE LAS LINEAS ESMALTADORAS Y DECORADORAS.
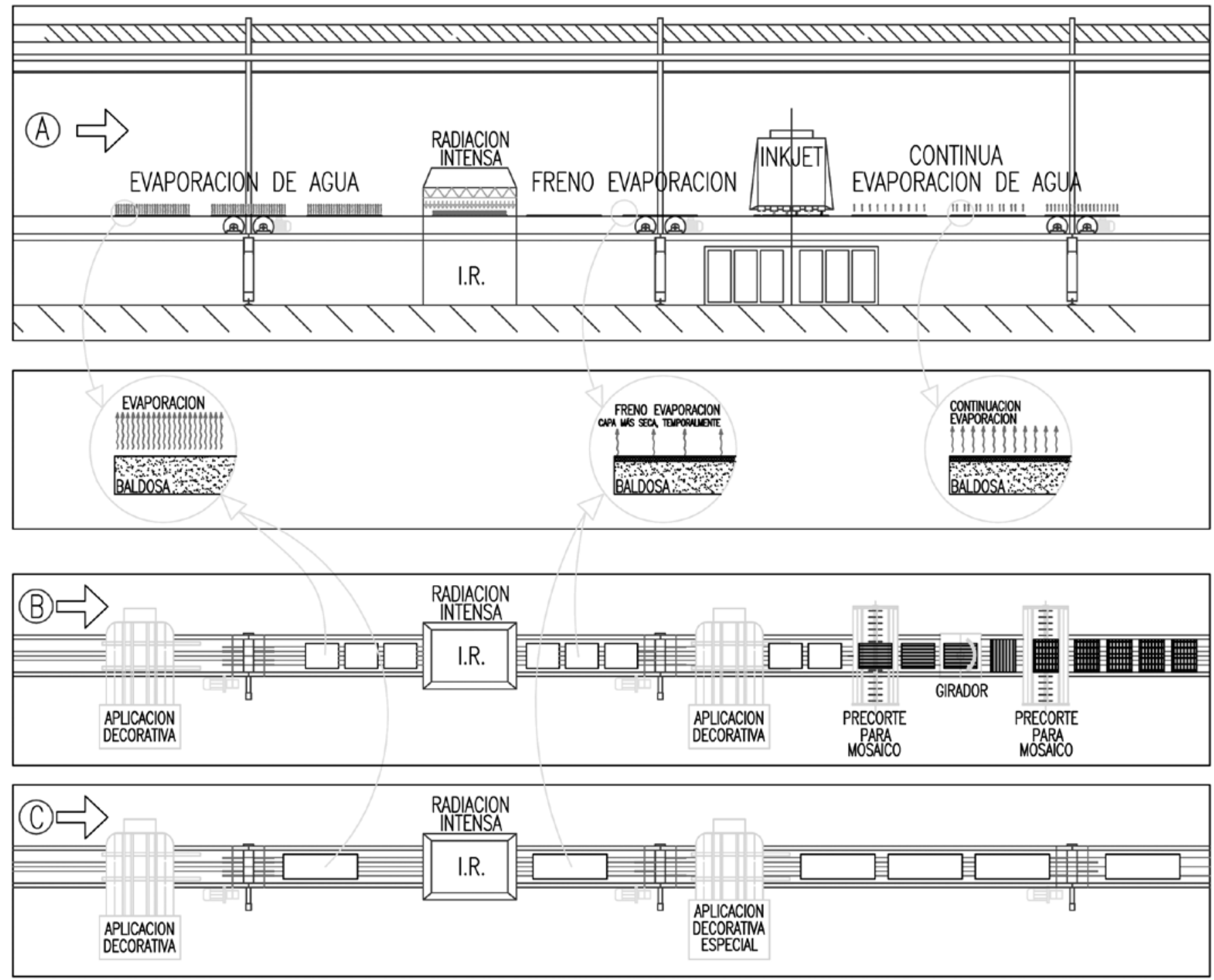
La solución se puede conseguir con una pequeña máquina denominada IR, que produce radiaciones sobre el material con longitud de onda regulable, en tiempo rapidísimo. Seguramente será la máquina más rápida existente en el mercado.

De este modo se pueden ajustar fácilmente las condiciones de trabajo a cada tipo de material y según las condiciones de temperatura y humedad de cada jornada a lo largo del año y para cada tipo de producto a decorar.

Manteniendo un estrato superficial con mínima humedad se obtiene:

- Una gota de tinta/esmalte, absorbida con nitidez por la baldosa cerámica.

- Una menor evaporación temporal de agua, y por tanto una protección de la impresora, aumentando la productividad, al ser menores las necesidades de parada para limpieza de inyectores.

\section{PRINCIPIO DE FUNCIONAMIENTO}

Equipo, que mediante aplicación de tecnología de longitud de onda (espectro electromagnético) permite un secado automático instantáneo sobre estratos y superficies húmedas, lo que posibilita una rápida manipulación de las baldosas en movimiento y tratamientos de decoración digital de gran nitidez.

Se puede combinar con sistema complementario de aire caliente, para conseguir efectos específicos.

\section{VENTAJAS QUE SE OBTIENEN CON EL EQUIPO}

a) Situada antes de las impresoras INKJET, evita condensaciones en los inyectores aumentando considerablemente la productividad de la línea de decoración.

b) Mejora la nitidez y calidad del diseño obtenido, aumentando el valor del producto.

\section{TECNOLOGIA DE LONGITUD DE ONDA}

\section{- ESCALA}


- ULTRAVIOLETA (UV) $180-400 \mathrm{~nm}$

- INFRARROJOS (IR) 700nm-1mm

- MICROONDAS (MW) $1 \mathrm{~mm}-1 \mathrm{~m}$ 
c) Situada después de la decoradora INKJET, permite secar totalmente las tintas aplicadas en soportes tipo slim de baja absorción; en diseños con mucha cantidad de tintas y en la aplicación sobre pieza fría (bicocción), que encharca la superficie de la pieza con pérdida de definición por el movimiento de la tinta.

Al secar totalmente la tinta y quedarse fijada; permite otras sucesivas aplicaciones: rodillos; protecciones; granillas, etc. que en otros casos quedan limitadas por la repulsión de las suspensiones acuosas y las tintas Inkjet que produce pérdida de definición de la gráfica.

d) Previa a precorte, de piezas de monococción esmaltadasdecoradas para formar mosaico, evita los defectos del esmalte muy húmedo que se adhiere al disco provocando pérdida de definición del corte y linealidad del mismo; así como los ensuciamientos superficiales debidos a las partículas despegadas del disco, cuando se adhiere esmalte húmedo.

e) Situada al final de línea de esmaltado, elimina la humedad del esmalte, evitando que se adhiera polvo del ambiente de modo irreversible, durante la etapa de almacenamiento en el parque de crudo. Este polvo a veces no se elimina por soplado antes del horno creando algunos defectos en la superficie del esmalte cocido.

f) Puede utilizarse para acelerar el curado de resinas en placas de cerámica o mármol, etc. g) Interrumpe $100 \%$ el consumo de energía, en las pausas de la producción.

h) No contamina, ni produce hollín. No produce emisiones atmosféricas de $\mathrm{CO} 2$ al no existir combustión.

i) Es fácilmente trasladable cuando sea preciso por que la línea se configure para otras decoraciones.

\section{BIBLIOGRAFÍA}

1. I. Hutchings. Qualicer 2010. Impresión por chorro de tinta para decoración de baldosas.

2. M. Alexander (2010). The Xaar guide to digital printing in the ceramic sector.

3. Vicente Lázaro, Ana Mateu, María Prada del I.T.C. /AICE Castellón. Técnica Cerámica nº 381, pág. 273 y 274

4. ESMALGLASS - ITACA. Esmaltes digitales para un proceso de esmaltación y decoración. Bol. Sociedad Española de Cerámica y Vidrio nº 50, Abril 2011.

5. Patente nacional $\mathrm{n}^{\circ}$ P200702733ES (en trámite internacional), de BULMA TECNOLOGÍA, S.L.

6. Infrarrojos, luz y ultravioletas. Núm. 113-Julio, Agosto 2002. Los trabajos prácticos de investigación. Revista Aula de Innovación educativa. Autor: Josep Corominas.

7. Guía de la termografía infrarroja. Aplicaciones en ahorro y eficiencia energética (2011) Autor: Sergio Melgosa 16/11/2011. 